\title{
Invasion threshold in heterogeneous metapopulation networks
}

\author{
Vittoria Colizza ${ }^{1}$ and Alessandro Vespignani ${ }^{2}$ \\ ${ }^{1}$ Complex Networks Lagrange Laboratory, Institute for Scientific Interchange, Torino 10133, Italy \\ ${ }^{2}$ Complex Systems Group, School of Informatics, Indiana University, Bloomington IN 47406
}

(Dated: October 25, 2018)

\begin{abstract}
We study the dynamics of epidemic and reaction-diffusion processes in metapopulation models with heterogeneous connectivity pattern. In SIR-like processes, along with the standard local epidemic threshold, the system exhibits a global invasion threshold. We provide an explicit expression of the threshold that sets a critical value of the diffusion/mobility rate below which the epidemic is not able to spread to a macroscopic fraction of subpopulations. The invasion threshold is found to be affected by the topological fluctuations of the metapopulation network. The presented results provide a general framework for the understanding of the effect of travel restrictions in epidemic containment.

PACS numbers: 89.75.-k, -87.23.Ge, 05.70Ln
\end{abstract}

The role of heterogeneity has been acknowledged as a central question in the study of population biology of infectious diseases [1, 2, 3] and revamped recently with the evidence that a large number of real world networks exhibit complex topological properties [4, 5, 6]. These features, often mathematically encoded in a heavy-tailed probability distribution $P(k)$ that any given node has degree $k$, were shown to affect the system evolution altering the threshold behavior and the associated dynamical phase transition $[7,8,8]$. These studies have mainly focused on networked systems where each node corresponds to a single individual and only recently the study of the impact of heterogeneous topologies on bosonic systems, where nodes can be occupied by any number of particles, has been initiated [10]. Examples are provided by reaction-diffusion systems used to model a wide range of phenomena in chemistry and physics 11], and metapopulation epidemic models [2, 3, 12, 13, 14, 15, 16] where particles represent people moving across different subpopulations (nodes) such as city or urban areas, and the reaction processes account for the local infection dynamics.

Here we analyze epidemic metapopulation models characterized by an infection dynamics within each node (or subpopulation) that follows a Susceptible-InfectedRemoved (SIR) model. The mobility rate $p$ of individuals defines the coupling process among the subpopulations. In the real world, the networks representing the mobility pattern of individuals among different subpopulations are in many cases highly heterogeneous 17, 18, 19, 20, 21. For this reason, the connectivity pattern of the metapopulation network is described as a random graph with arbitrary degree distribution $P(k)$. By using a mechanistic approach it is possible to show that along with the usual epidemic threshold condition $R_{0}>1$ on the basic reproductive number, the system exhibits a global invasion threshold setting the condition for the infection of a macroscopic fraction of the metapopulation system [26, 27]. The threshold condition on $R_{0}$ ensures the local outbreak at the subpopulation level [1, 10], whereas the explicit expression obtained for global invasion threshold $R_{*}>1$ provides a critical value for the diffusion rate $p$ below which the epidemic cannot propagate to a relevant fraction of subpopulations. We find that the global invasion threshold is affected by the topological fluctuations of the underlying network. The larger the network heterogeneity and the smaller is the value of the critical diffusion rate above which the epidemic may globally invade the metapopulation system. The present results can be generalized to more realistic diffusion and mobility schemes and provide a framework for the analysis of realistic metapopulation epidemic models [22, 23, 24, 25, 28].

A simplified mechanistic (i.e. microscopic in the epidemic terminology) approach to the metapopulation spreading of infectious diseases uses a markovian assumption in which at each time step the movement of individuals is given in terms of a matrix $d_{i j}$ that expresses the probability that an individual in the subpopulation $i$ is traveling to the subpopulation $j$. Several modeling approaches to the large scale spreading of infectious diseases [22, 23, 24, 25, 29] use this mobility process based on transportation networks combined with the local evolution of the disease. The markovian character lies in the assumption that at each time step the same traveling probability applies to all individuals in the subpopulation without having memory of their origin. This mobility scheme coupled with an infection dynamics at the local level can be generally viewed as equivalent to classic reaction diffusion processes with no constraint on the occupation numbers $N_{i}$ of each subpopulation. The total population of the metapopulation system is $N=\sum_{i} N_{i}$ and each individual diffuses along the edges with a diffusion coefficient $d_{i j}$ that depends on the node degree, subpopulation size and/or the mobility matrix. The metapopulation system is therefore composed of a network substrate connecting nodes - each corresponding to a subpopulation - over which individuals diffuse. We consider that 
each node $i$ is connected to other $k_{i}$ nodes according to its degree resulting in a network with degree distribution $P(k)$ and distribution moments $\left\langle k^{\alpha}\right\rangle=\sum_{k} k^{\alpha} P(k)$.

In the following, as a simplified diffusion process we assume that the mobility is equivalent to a diffusion rate along any given link of a node with degree $k$ simply equal to $d_{k k^{\prime}}=p / k$. This is obviously not the case in a wide range of real systems where the extreme heterogeneity of traffic is well documented and more realistic processes will be considered elsewhere. This simple process however automatically generates a stationary distribution of occupation numbers that is better described by grouping subpopulations according to their degree $k$

$$
N_{k}=\frac{k}{\langle k\rangle} \bar{N}
$$

where $\bar{N}$ is the average subpopulation size.

In each subpopulation $j$ the disease follows an SIR model and the total number of individuals is partitioned in the compartments $S_{j}(t), I_{j}(t)$ and $R_{j}(t)$, denoting the number of susceptible, infected and removed individuals at time $t$, respectively. The infection dynamics proceeds as follows. Each susceptible individual has a transition rate to the infected state expressed as $\beta I_{j} / N_{j}$, where $\beta$ is the disease transmissibility rate and $I_{j} / N_{j}$ is the force of infection in the homogeneous mixing assumption. Analogously, each infected individual enters the removed compartment according to the recovery rate $\mu$. The basic SIR rules thus define a reaction scheme of the type $S+I \rightarrow 2 I$ and $I \rightarrow R$, that conserves the number of individuals. The SIR epidemic model is characterized by the reproductive number $R_{0}=\beta / \mu$ that defines the average number of infectious individuals generated by one infected individual in a fully susceptible population. The epidemic is able to generate a number of infected individuals larger than those who recover only if $R_{0}>1$, yielding the classic result for the epidemic threshold [1]. If the spreading rate is not large enough to allow a reproductive number larger than one (i.e. $\beta>\mu$ ), the epidemic outbreak will quickly die out. This result is valid at the level of each subpopulation and holds also at the metapopulation level where $R_{0}>1$ is a necessary condition to have the growth of the epidemic [10].

The intuitive result on the subpopulation epidemic threshold however does not take into account the effects due to the finite size of subpopulations, the discrete nature of individuals and the stochastic nature of the reaction and diffusion processes. These effects have been shown to have a crucial role in the problem of resurgent epidemics, extinction and eradication [26, 27, 30, 31]. Also in the present framework indeed each subpopulation may or may not transmit the infection to a neighboring subpopulation upon the condition that at least one infected individual is moving onto the non-infected subpopulations during the epidemic outbreak. Given an
SIR model with $R_{0}>1$, the total number of infected individuals generated within a subpopulation and the mobility rate must be large enough to ensure the seeding of other subpopulations before the end of the local outbreak [26, 27].

As a simple example of this effect let us consider a metapopulation system in which the initial condition is provided by a single infection in a subpopulation with degree $k$ and $N_{k}$ individuals, given $R_{0}>1$. In the case of a macroscopic outbreak in a closed population the total number of infected individuals during the outbreak evolution will be equal to $\alpha N_{k}$ where $\alpha$ depends on the specific disease model and parameter values used. Each infected individual stays in the infectious state for an average time $\mu^{-1}$ equal to the inverse of the recovery rate, during which it can travel to the neighboring subpopulation of degree $k^{\prime}$ with rate $d_{k k^{\prime}}$. We can therefore consider that on average the number of new seeds that may appear into a connected subpopulation of degree $k^{\prime}$ during the duration of the local outbreak is given by

$$
\lambda_{k k^{\prime}}=d_{k k^{\prime}} \frac{\alpha N_{k}}{\mu} .
$$

In this perspective we can provide a characterization of the invasion dynamics at the level of the subpopulations, translating epidemiological and demographic parameters into Levins-type metapopulation parameters of extinction and invasion rate. Let us define $D_{k}^{0}$ as the number of diseased subpopulation of degree $k$ at generation 0 , i.e. those which are experiencing an outbreak at the beginning of the process. Each infected subpopulation will seed - during the course of the outbreak - the infection in neighboring subpopulations defining the set $D_{k}^{1}$ of infected subpopulations at generation 1, and so on. This corresponds to a basic branching process [26, 32, 33] where the $n$-th generation of infected subpopulations of degree $k$ is denoted $D_{k}^{n}$.

In order to describe the early stage of the subpopulations invasion dynamics we assume that the number of subpopulations affected by an outbreak (with $R_{0}>1$ ) is small and we can therefore study the evolution of the number of diseased subpopulations by using a branching process approximation relating $D_{k}^{n}$ with $D_{k}^{n-1}$. Let us consider a metapopulation network with degree distribution $P(k)$ and $V$ subpopulations and write the number of subpopulations of degree $k$ invaded at the generation $n$ as:

$D_{k}^{n}=\sum_{k^{\prime}} D_{k^{\prime}}^{n-1}\left(k^{\prime}-1\right)\left[1-\left(\frac{1}{R_{0}}\right)^{\lambda_{k^{\prime} k}}\right] P\left(k \mid k^{\prime}\right)\left(1-\frac{D_{k}^{n-1}}{V_{k}}\right)$.

This equation assumes that each infected subpopulation of degree $k^{\prime}$ of the $(n-1)$-th generation, $D_{k^{\prime}}^{n-1}$, will seed the infection in a number $\left(k^{\prime}-1\right)$ of subpopulations corresponding to the number of neighboring subpopulations $k^{\prime}$ minus the one which originally transmitted the 
infection, the probability $P\left(k \mid k^{\prime}\right)$ that each of the $k^{\prime}-1$ not yet infected neighboring subpopulations has degree $k$, and the probability to observe an outbreak in the seeded subpopulation i.e. $\left(1-R_{0}^{-\lambda_{k k}}\right)$ [36]. The last factor stems from the probability of extinction $P_{\text {ext }}=1 / R_{0}$ of an epidemic seeded with a single infectious individual [36]. In order to obtain an explicit result we will consider in the following that $R_{0}-1 \ll 1$, thus assuming that the system is found to be very close to the epidemic threshold. In this limit we can approximate the outbreak probability as $1-R_{0}^{-\lambda_{k^{\prime} k}} \simeq \lambda_{k^{\prime} k}\left(R_{0}-1\right)$. The case of homogeneous diffusion of individuals $d_{k}=p / k$ with the stationary solution of eq. (2) for the subpopulation size yields $\lambda_{k^{\prime} k}=p \bar{N} \alpha \mu^{-1} /\langle k\rangle$. In addition, we assume that at the early stage of the epidemic $D_{k}^{n-1} / V_{k} \ll 1$, and we consider the case of uncorrelated networks in which the conditional probability does not depend on the originating node, i.e. $P\left(k \mid k^{\prime}\right)=k P(k) /\langle k\rangle[5]$, obtaining

$$
D_{k}^{n}=\left(R_{0}-1\right) \frac{k P(k)}{\langle k\rangle^{2}} \frac{p \bar{N} \alpha}{\mu} \sum_{k^{\prime}} D_{k^{\prime}}^{n-1}\left(k^{\prime}-1\right) .
$$

By defining $\Theta^{n}=\sum_{k^{\prime}} D_{k^{\prime}}^{n}\left(k^{\prime}-1\right)$ the last expression can be conveniently written in the iterative form

$$
\Theta^{n}=\left(R_{0}-1\right) \frac{\left\langle k^{2}\right\rangle-\langle k\rangle}{\langle k\rangle^{2}} \frac{p \bar{N} \alpha}{\mu} \Theta^{n-1},
$$

that allows the increasing of infected subpopulations and a global epidemic in the metapopulation process only if

$$
R_{*}=\left(R_{0}-1\right) \frac{\left\langle k^{2}\right\rangle-\langle k\rangle}{\langle k\rangle^{2}} \frac{p \bar{N} \alpha}{\mu}>1,
$$

defining the global invasion threshold of the metapopulation system. In other words, $R_{*}$ is the analogous of the basic reproductive number at the subpopulations level and is a crucial indicator in assessing the behavior of epidemics in metapopulation models. Its expression indeed contains the probability of generating an outbreak in a neighbor subpopulation by means of mobility processes, $\left(R_{0}-1\right) p \bar{N} \alpha /(\mu\langle k\rangle)$ for $R_{0}-1 \ll 1$, times the factor $\left\langle k^{2}\right\rangle /\langle k\rangle-1$ which also appears in the threshold conditions characterizing phase transitions on complex networks [7, 33, 34, 35]. The explicit form of Eq. (6) can be used to find the minimum mobility rate ensuring that on average each subpopulation can seed more than one neighboring subpopulations. The constant $\alpha$ is larger than zero for any $R_{0}>1$, and in the SIR case for $R_{0}$ close to 1 it can be approximated by $\alpha \simeq 2\left(R_{0}-1\right) / R_{0}^{2}[36]$, yielding for the SIR model a critical mobility value $p_{c}$ below which the epidemics cannot invade the metapopulation system given by the equation

$$
p_{c} \bar{N}=\frac{\langle k\rangle^{2}}{\left\langle k^{2}\right\rangle-\langle k\rangle} \frac{\mu R_{0}^{2}}{2\left(R_{0}-1\right)^{2}},
$$

The above condition readily tells us that the closer to the epidemic threshold is the single subpopulation outbreak

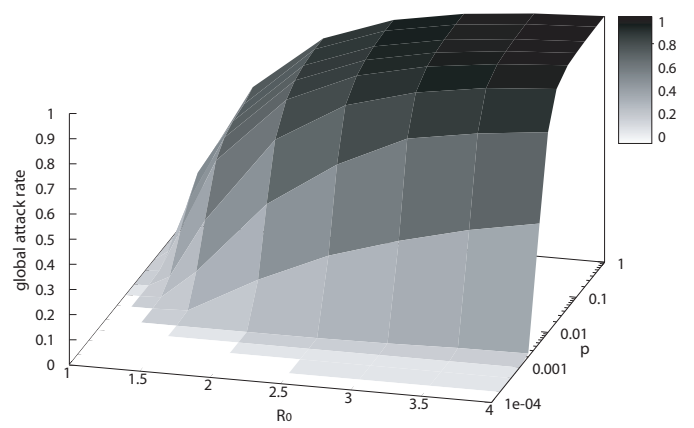

FIG. 1: Phase diagram of the metapopulation system. The final epidemic size is shown as a function of the local threshold $R_{0}$ and of the diffusion probability $p$.

and the larger it has to be the mobility rate in order to sustain the global spread into the metapopulation model. It is important to stress that when $R_{0}$ increases, the small $\left(R_{0}-1\right)$ expansions are no longer valid and the invasion threshold is obtained only in the form of a complicate implicit expression.

In addition, eq. (7) shows the dependance of the critical mobility on the topological fluctuations of the mobility network. The ratio $\langle k\rangle^{2} /\langle k(k-1)\rangle$ is extremely small in heavy-tailed networks and it is vanishing in the limit of infinite network size. This implies that the heterogeneity of the metapopulation network is favoring the global spread of epidemics by lowering the global spreading threshold. In other words, the topological fluctuations favor the subpopulation invasion and suppress the phase transition in the infinite size limit. This finding provides a theoretical framework and rationale for the evidence concerning the inefficacy of travel restrictions in the containment of global epidemics [25, 38]. The simple plug in of the actual numbers for modern transportation networks, the population sizes and realistic disease parameters in the expression (7) indicates that a reduction of one order of magnitude of the mobility is not enough to bring the system below the invasion threshold. While more complicate mobility schemes should be considered for a precise calculation, this result is setting the framework for the understanding of mobility effects in the spreading and containment of infectious diseases.

In order to support the previous analytical finding we have performed an extensive set of Monte Carlo numerical simulations of the metapopulation system. The substrate network is given by an uncorrelated complex network with $P(k) \sim k^{-2.1}$ generated with the uncorrelated configuration model [37] to avoid inherent structural correlations. Network sizes of $V=10^{4}$ and $10^{5}$ nodes have been considered. The dynamics proceeds in parallel and considers discrete time steps representing the unitary time scale $\tau$ of the process. The reaction and diffusion rates are therefore converted into probabilities and 
at each time step in each subpopulation $j$ a susceptible individual is turned into an infectious with probability $1-\left(1-\frac{\beta}{N_{j}} \tau\right)^{I_{j}}$ and each infectious individual is subject to the recovery process and becomes recovered with probability $\mu \tau$. The mobility is modeled assuming a diffusion probability for each individual along each link of the subpopulation of the form $d_{k k^{\prime}}=p / k$.

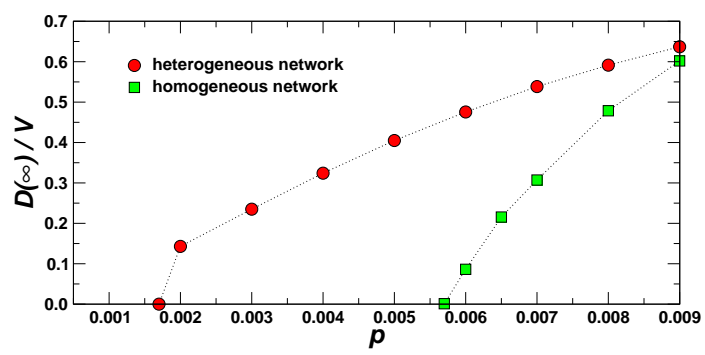

FIG. 2: Effect of the network heterogeneity on the global epidemic threshold. The final fraction of diseased subpopulations $D(\infty) / V$ at the end of the global epidemic is shown as a function of the mobility rate $p$ in a homogeneous and a heterogeneous network.

A complete analysis of the system phase diagram is obtained by analyzing the behavior of the global attack rate $R(\infty) / N$, defined as the total fraction of cases in the metapopulation system at the end of the epidemic, as a function of both $R_{0}$ and $p$. Figure 1 reports the global attack rate surface in the $p-R_{0}$ space and clearly shows the effect of different couplings as expressed by the value of $p$ in reducing the final size of the epidemic at a given fixed value of $R_{0}$. The smaller the value of $R_{0}$, the higher the coupling needs to be in order for the virus to successfully invade a finite fraction of the subpopulations, in agreement with the analytic result of eq. (7). This provides a clear illustration of the varying global invasion threshold as a function of the reproductive rate $R_{0}$. Furthermore, it is possible to study the effect of the heterogeneity of the metapopulation structure on the global epidemic threshold. Figure 2 shows the results obtained by comparing two random metapopulations networks, one with poissonian degree distribution (homogeneous network) and one with heavy-tailed $\left(P(k) \sim k^{-2.1}\right)$ degree distribution (heterogeneous neywork). Despite the two models have the same average degree, disease parameters, the fluctuations of the power-law network increase the value of $R_{*}$ thus lowering the critical value of the mobility.

The present analysis provides insights in setting a framework for the analysis of large scale spreading of epidemics in realistic mobility networks. Furthermore, these results open the path to future work aimed at analyzing refined metapopulation infection models.

A. V. is partially funded by the NSF IIS-0513650. V.C. and A.V. are partially funded by the CRT foundation.
[1] R.M. Anderson and R.M. May, Infectious diseases in humans (Oxford University Press, Oxford 1992).

[2] H.W. Hethcote, Theor. Pop. Biol. 14, 338 (1978).

[3] R.M. May and R.M. Anderson, Math. Biosci 72, 83 (1984).

[4] R. Albert and A.-L. Barabási, Rev. Mod. Phys. 74, 47 (2000).

[5] S. N. Dorogovtsev and J. F. F. Mendes, Evolution of networks (Oxford University Press, Oxford 2003).

[6] M.E.J. Newman, SIAM Rev. 45, 167 (2003).

[7] R. Pastor-Satorras and A. Vespignani, Phys. Rev. Lett. 86, 3200 (2001).

[8] A.L. Lloyd and R.M. May, Science 292, 1316 (2001).

[9] M. Barthélemy et al., J. Theor. Biol. 235, 275 (2005).

[10] V. Colizza, R. Pastor-Satorras and A. Vespignani, Nature Phys. 3, 276 (2007).

[11] N.G. van Kampen, Stochastic Processes in Chemistry and Physics (Springer, Berlin, 2005).

[12] M.J. Keeling and P. Rohani, Ecol. Lett. 5, 20 (2002).

[13] A. L. Lloyd and R. M. May, J. Theor. Biol. 179, 1 (1996).

[14] B.T. Grenfell and J. Harwood, TREE 12, 395 (1997).

[15] B.T. Grenfell and B.M. Bolker, Ecol Lett. 1, 63 (1998).

[16] N. M. Ferguson et al., Nature 425, 681 (2003).

[17] G. Chowell et al., Phys. Rev. E 68, 066102 (2003).

[18] C. L. Barrett et al., Technical Report LA-UR-00-1725, Los Alamos National Laboratory (2000).

[19] A. Barrat et al. Proc. Natl. Acad. Sci. USA 101, 3747 (2004).

[20] R. Guimerá et al., Proc. Natl. Acad. Sci. USA 102, 7794 (2005).

[21] A. De Montis et al., Env. Planning B doi:10.1068/b32128 (2007).

[22] R.F. Grais, J.H. Ellis and G.E. Glass, Eur. J. Epidemiol. 18, 1065 (2003).

[23] L. Hufnagel, D. Brockmann and T. Geisel, Proc. Natl. Acad. Sci. USA 101, 15124 (2004).

[24] V. Colizza et al., Proc. Natl. Acad. Sci. USA 103, 2015 (2006).

[25] V. Colizza et al., PLoS Med. 4(1), e13 (2007).

[26] F. Ball, D. Mollison, G. Scalia-Tomba, Ann. Appl. Probab. 7, 46 (1997).

[27] P. Cross et al., Ecol. Lett. 8, 587 (2005).

[28] S. Riley, Science 316, 1298 (2007).

[29] L.A. Rvachev and I.M. Longini, Math. Biosci. 75, 3 (1985).

[30] D. Watts et al., Proc. Natl. Acad. Sci. USA 102, 11157 (2005).

[31] A. Vázquez, J. Theor. Biol. 245, 125 (2007).

[32] T.E. Harris, The theory of branching processes Dover Publications (1990).

[33] A. Vázquez, Phys. Rev. Lett. 96, 038702 (2006).

[34] D.S. Callaway et al., Phys. Rev. Lett. 85, 5468 (2000).

[35] R. Cohen et al. Phys. Rev. Lett. 85, 4626 (2000).

[36] N.T. Bailey, The Mathematical Theory of Infectious Diseases (2nd edition, Hodder Arnold, 1975).

[37] M. Catanzaro, M. Boguña and R. Pastor-Satorras, Phys. Rev. E 71, 027103 (2005).

[38] T.D. Hollingsworth, N.M. Ferguson and R.M. Anderson, Nature Med. 12, 497 (2006). 